\title{
Radio Observations of Star Forming Galaxies in the SKA era
}

\author{
Claudia Mancuso*1 ${ }^{*}$ Andrea Lapi ${ }^{1,2}$, Zhen-Yi Cai ${ }^{3}$, Mattia Negrello ${ }^{4}$, Gianfranco De \\ Zotti $^{1,4}$, Francesca Perrotta ${ }^{1}$, Luigi Danese ${ }^{1}$ \\ ${ }^{1}$ Astrophysics Sector, SISSA, Via Bonomea 265, I-34136 Trieste ; ${ }^{2}$ Dipartimento di Fisica, \\ Università 'Tor Vergata', Via della Ricerca Scientifica 1, I-00133 Roma, Italy ; ${ }^{3}$ Center for \\ Astrophysics, University of Science and Technology of China, Hefei, 230026, China; ${ }^{4}$ INAF - \\ Osservatorio Astronomico di Padova, Vicolo dell'Osservatorio 5, I-35122 Padova, Italy \\ E-mail:cmancusoesissa.it
}

\begin{abstract}
We have combined determinations of the epoch-dependent star formation rate (SFR) function with relationships between SFR and radio (synchrotron and free-free) emission to work out detailed predictions for the counts and the redshift distributions of star-forming galaxies detected by planned Square Kilometer Array (SKA) surveys. The evolving SFR function comes from recent models fitting the far-infrared (FIR) to millimeter-wave luminosity functions and the ultraviolet (UV) luminosity functions up to $z=10$, extended to take into account additional UV survey data. We used very deep $1.4 \mathrm{GHz}$ number counts from the literature to check the relationship between SFR and synchrotron emission, and the $95 \mathrm{GHz}$ South Pole Telescope (SPT) counts of dusty galaxies to test the relationship between SFR and free-free emission. We show that the SKA will allow us to investigate the SFRs of galaxies down to few $M_{\odot} / \mathrm{yr}$ up to $\mathrm{z}=10$, thus extending by more than two orders of magnitude the high-z SFR functions derived from Herschel surveys. SKA1-MID surveys, down to $\mu \mathrm{Jy}$ levels, will detect hundreds of strongly lensed galaxies per square degree; a substantial fraction of them will show at least two images above the detection limits.
\end{abstract}

Advancing Astrophysics with the Square Kilometre Array

June 8-13, 2014

Giardini Naxos, Italy

\footnotetext{
* Speaker.
} 


\section{Introduction}

The huge amount of infrared (IR) to millimeter-wave data that has been accumulating in the last several years thanks to Spitzer, Herschel, SCUBA and South Pole Telescope (SPT) surveys has made possible spectacular advances in our understanding of galaxy evolution. In fact the interstellar dust absorbs and re-emits at IR wavelengths about half of the starlight of the Universe (Casey et al., 2014). Hence, the evolution of the IR luminosity function directly maps that of the dust-enshrouded star formation rate (SFR). Herschel data are particularly powerful in this respect as they probe the dust emission peak, thus providing the best estimates of the total IR luminosity.

However, the IR emission misses the starlight not absorbed by dust and therefore underestimates the SFR whenever the absorption optical depth is not very high. This is the case for the earliest phases of galaxy evolution when the metal enrichment of the interstellar medium is just beginning, as well as for dust-poor dwarf galaxies and metal-poor regions of more-massive galaxies (Kennicutt \& Evans, 2012). A complete inventory of the SFR requires a combination of IR and UV photometry and thus suffers from limitations in both wavebands.

An important alternative tool for measuring the cosmic star formation history of the Universe is provided by deep radio continuum surveys. A tight relationship between low-frequency radio (synchrotron) and IR luminosity (hence SFR) has long been established (Helou, Soifer, \& RowanRobinson, 1985; Condon, 1992; Yun, Reddy, \& Condon, 2001; Ivison et al., 2010; Jarvis et al., 2010; Bourne et al., 2011; Mao et al., 2011). However the physical basis of this relationship is not yet totally clear. In fact many physical processes (propagation of relativistic electrons, strength and structure of the magnetic field, size and composition of dust grains) must conspire together to produce this relation (Bell, 2003; Helou et al., 1993; Niklas et al., 1997; Murphy, 2009; Lacki et al., 2010; Hippelein et al., 2003). Hence it is not granted that the relation also applies to redshift/luminosity ranges where the available data are insufficient to test it accurately. Moreover we cannot be sure that the observed synchrotron emission is not contaminated by faint nuclear activity.

There is a second process contributing to the radio emission of star-forming galaxies: the freefree emission from hot electrons, which is directly proportional to the production rate of ionising photon by young, massive stars. It shows up at rest frame frequencies of tens of $\mathrm{GHz}$, where it is generally optically thin, and thus offers a clean way to quantify the current star formation activity in galaxies. This picture could be complicated by the presence of anomalous dust emission (Planck Collaboration XX, 2011, and references therein) which occurs at similar frequencies and is thought to arise from spinning dust grains (e.g., Draine \& Lazarian, 1998). However there is currently no evidence that this component contributes significantly to globally integrated measurements (Murphy et al., 2012). Thus high frequency radio observations may be particularly powerful for precisely measuring the star formation history of the Universe.

Very deep radio surveys have shown that, at GHz frequencies, the counts below 100-200 $\mu \mathrm{Jy}$ are dominated by star-forming galaxies (Padovani et al., 2011, and references therein). Current surveys only extend to a few tens of $\mu \mathrm{Jy}$, i.e. cover a flux density range where, at low radio frequencies, the detected radio emission is of synchrotron origin. Only with the advent of the Square Kilometer Array (SKA) we expect that the high- $z$ star-forming galaxies can be seen via their free-free emission (see also Murphy, E. J., "The Astrophysics of Star Formation Across Cosmic Time at $\mathrm{z} \geq 10 \mathrm{GHz}$ with the Square Kilometre Array", PoS (AASKA14)085). 
In this paper we carry out a thorough investigation of the radio counts of star forming galaxies. In Sect. 2 we present a short outline of the model, that builds on the work by Cai et al. (2013) and Cai et al. (2014). In Sect. 3 we discuss the calibration of the relation between radio emissions and SFR. The South Pole Telescope (SPT) surveys at mm wavelengths are especially useful to test the SFR/free-free relation; this motivated a re-analysis of the SPT $95 \mathrm{GHz}$ sample of dusty galaxies. These relations allow us to extend to radio frequencies the Cai et al. (2013) model for the cosmological evolution of star forming galaxies and exploit it, in Sect. 4, to work out predictions for the counts of such galaxies in the range $1.4-30 \mathrm{GHz}$. We also compare the coverage of the SFR $-z$ plane by Herschel, UV surveys and SKA. Finally, Sect. 5 summarizes our main conclusions. Predictions of number counts of galaxies based on a different approach are presented by Jarvis, M., "Radio Continuum Simulations for the SKA", PoS (AASKA14)080.

Throughout this paper we adopt a flat $\Lambda \mathrm{CDM}$ cosmology with matter density $\Omega_{\mathrm{m}}=0.32$, $\Omega_{\mathrm{b}}=0.049, \Omega_{\Lambda}=0.68$, Hubble constant $h=H_{0} / 100 \mathrm{~km} \mathrm{~s}^{-1} \mathrm{Mpc}^{-1}=0.67$, spectrum of primordial perturbations with index $n=0.96$ and normalization $\sigma_{8}=0.83$ (Planck Collaboration XVI, 2013).

\section{Outline of the model}

A direct tracer of recent star formation is the UV emission of galaxies, coming from the photospheric emission of massive young stars. In recent years a great effort has been made to measure the UV luminosity functions up to high redshifts (Bouwens et al., 2008, 2011; Smit et al., 2012; Oesch et al., 2012, 2013a,b; Schenker et al., 2013; McLure et al., 2013), with the aim of reconstructing the history of cosmic re-ionization.

However, as the chemical enrichment of the ISM proceeds and, correspondingly, the dust abundance increases, a larger and larger fraction of starlight is absorbed and re-emitted at far-IR wavelengths. The most active star-formation phases of high- $z$ galaxies indeed suffer by strong dust obscuration and are most effectively studied in the far-IR/sub-mm region.

A comprehensive investigation of the evolution of the IR luminosity functions has been recently carried out by Cai et al. (2013) based on a "hybrid" approach that reflects the observed dichotomy in the ages of stellar populations of early-type galaxies on one side and late-type galaxies on the other (cf. Bernardi et al., 2010, their Fig. 10). Early-type galaxies and massive bulges of Sa galaxies are composed of relatively old stellar populations with mass-weighted ages $\gtrsim 8-9 \mathrm{Gyr}$ (corresponding to formation redshifts $z \gtrsim 1-1.5$ ), while the disk components of spirals and the irregular galaxies are characterized by significantly younger stellar populations. Thus the progenitors of early-type galaxies, referred to as proto-spheroidal galaxies or protospheroids, are the dominant star-forming population at $z \gtrsim 1.5$, while IR galaxies at $z \lesssim 1.5$ are mostly late-type "cold" (normal) and "warm" (starburst) galaxies.

The Cai et al. (2013) model accurately fits a broad variety of data ${ }^{1}$ : multi-frequency and multiepoch luminosity functions of galaxies and AGNs, redshift distributions, number counts (total and per redshift bins). Moreover, it accurately accounts for the recently determined counts and redshift distribution of strongly lensed galaxies detected by the South Pole Telescope (SPT; Mocanu et al., 2013; Weiß et al., 2013), published after the paper was completed (Bonato et al., 2014).

\footnotetext{
${ }^{1}$ See figures in http://people.sissa.it/ zcai/galaxy_agn/.
} 
In general the total $(8-1000 \mu \mathrm{m})$ IR luminosity, $L_{\mathrm{IR}}$, is a good proxy of the obscured SFR when the dust heating is dominated by young stars. In disks of normal galaxies, however, the IR luminosity is the sum of a "warm" component heated by young stars and of a "cold" (or "cirrus") component, heated by the general radiation field that may be dominated by older stars. This issue was investigated by Clemens et al. (2013) using a complete sample of local star-forming galaxies detected by Planck. We adopt the relation between SFR and $L_{\mathrm{IR}}$ derived by these authors, for a Chabrier initial mass function (IMF).

As mentioned in Sect. 1, to get a complete census of the cosmic SFR we need to complement IR measurements with UV SFR tracers to measure the unattenuated starlight. For the high- $z$ protospheroidal galaxies we adopted the accurately tested physical model for the evolution of the UV luminosity function worked out by Cai et al. (2014) in the framework of the scenario proposed by Granato et al. (2004) and further elaborated by Lapi et al. (2006, 2011), Mao et al. (2007), and Cai et al. (2013).

For the low- $z$ galaxies we have supplemented the Cai et al. (2013) model for evolution of late-type galaxies in the IR with a parametric model for evolution in the UV. Briefly, the UV $(\lambda=1500 \AA)$ luminosity function is described by:

$$
\Phi\left(\log L_{1500}, z\right) \mathrm{d} \log L_{1500}=\Phi^{*}\left(\frac{L_{1500}}{L^{*}}\right)^{1-\alpha} \times \exp \left[-\frac{\log ^{2}\left(1+L_{1500} / L^{*}\right)}{2 \sigma^{2}}\right] \mathrm{d} \log L_{1500} .
$$

A simple pure luminosity evolution model $\left(L^{*}(z)=L_{0}^{*}(1+z)^{\alpha_{L}}\right.$ up to $\left.z=1\right)$ turned out to provide a sufficiently good description of the data (Mancuso et al., in preparation). The best-fit values of the parameters are $\log \left(\Phi_{0}^{*} /\left[\mathrm{dex}^{-1} \mathrm{Mpc}^{-3}\right]\right)=-2.150 \pm 0.095, \log \left(L_{0}^{*} / L_{\odot}\right)=9.436 \pm 0.119$, $\alpha=1.477 \pm 0.050, \sigma=0.326 \pm 0.035$, and $\alpha_{L}=2.025 \pm 0.063$.

The Kennicutt \& Evans (2012) calibrations were adopted to convert the UV luminosity functions into SFR functions. The total redshift-dependent SFR functions were then computed summing the IR-based and the UV-based ones.

\section{Calibration of the relation between radio emission and SFR}

A tight linear correlation between the $1.4 \mathrm{GHz}$ luminosity, dominated by synchrotron, and the IR luminosity has been established since many years (Condon, 1992). A calibration of the relation between the SFR and the synchrotron emission was calculated by Murphy et al. (2011). In order to take into account electron ageing effects (Banday \& Wolfendale, 1991) we have adopted a steepening by $\Delta \alpha=0.5$ above a break frequency of $20 \mathrm{GHz}$. The relationship between synchrotron luminosity and SFR then writes:

$$
L_{\mathrm{sync}} \simeq 1.9 \times 10^{28}\left(\frac{\mathrm{SFR}}{\mathrm{M}_{\odot} \mathrm{yr}^{-1}}\right)\left(\frac{v}{\mathrm{GHz}}\right)^{-0.85}\left[1+\left(\frac{v}{20 \mathrm{GHz}}\right)^{0.5}\right]^{-1} \mathrm{erg} \mathrm{s}^{-1} \mathrm{~Hz}^{-1} .
$$

Coupling this relation with the redshift dependent SFR functions yielded by the model outlined in Sect. 2 we get a good fit to the sub-mJy $1.4 \mathrm{GHz}$ counts (Fig. 1, left panel) without any adjustment of the parameters. The high-frequency synchrotron emission is increasingly suppressed with increasing $z$, as the timescale for energy losses of relativistic electrons by inverse Compton scattering off the Cosmic Microwave Background photons decreases as $(1+z)^{-4}$ (Norris et al., 2013; Carilli 

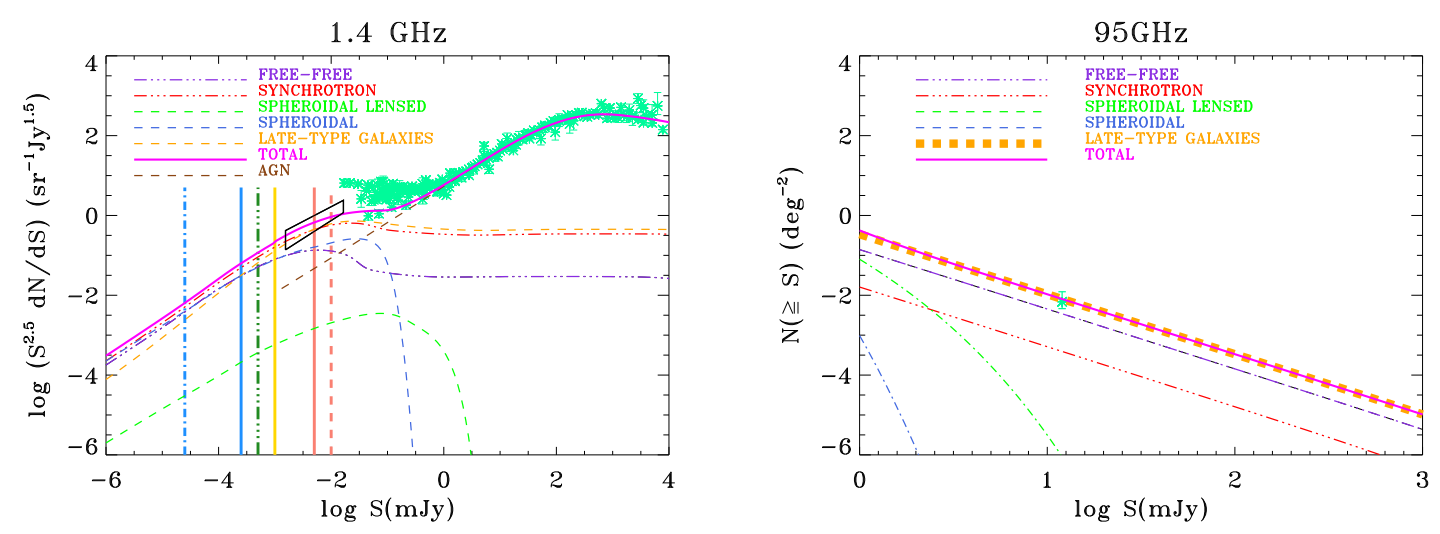

Figure 1: left panel: Euclidean normalized differential counts at $1.4 \mathrm{GHz}$ compared with model predictions. The dashed brown line shows the best fit model for radio AGNs by Massardi et al. (2010). The other dashed lines show the contributions of the star-forming populations considered in this paper. The two triple-dot dashed lines show the total synchrotron and free-free emissions from these populations. The solid vertical lines correspond, from left to right, to three $5 \sigma$ detection limits of preliminarily planned SKA1-MID surveys. The vertical blue dash-dotted line and the pink dashed line are respectively the flux limits for Ultra-Deep SKA2 (MID) and for the 50\% Wide SKA1-MID surveys, while the green dash-dot-dotted line is the limit for both 50\% Deep SKA1-MID and Wide SKA2 (MID) surveys. Right panel: our re-assessment of the integral counts of dusty galaxies at $v=95 \mathrm{GHz}$ (asterisks with error bars) compared with the expectations from the Cai et al. (2013) model.

et al., 2008; Murphy, 2009). This may lower the counts at the faintest flux densities, but not by a large factor since, as illustrated by Fig. 1 (left panel), below the limits of current surveys the free-free contribution is comparable to the synchrotron one.

A relationship between SFR and free-free emission was derived by Murphy et al. (2012). We have reformulated it as:

$$
L_{\mathrm{ff}}=3.75 \times 10^{26}\left(\frac{\mathrm{SFR}}{M_{\odot} / \mathrm{yr}}\right)\left(\frac{T}{10^{4} \mathrm{~K}}\right)^{-0.5} \mathrm{~g}(v, \mathrm{~T}) \exp \left(-\frac{h v}{k \mathrm{~T}}\right) \mathrm{erg} \mathrm{s}^{-1} \mathrm{~Hz}^{-1}
$$

where $\mathrm{T}$ is the temperature of the emitting plasma and $\mathrm{g}(v, \mathrm{~T})$ is the Gaunt factor for which we adopt the approximation proposed by Draine (2011) which is more accurate than the one used by Murphy et al. (2012). The coefficient of eq. (3.2) was computed requiring that this equation equals that by Murphy et al. (2012) for $v=33 \mathrm{GHz}$ (the frequency at which the relation was calibrated), $T=10^{4} \mathrm{~K}$ and a pure hydrogen plasma.

We note that the calibrations of the above relationships are based on the Kroupa IMF while that between the IR emission and the SFR (Sect. 2) relies on the Chabrier IMF. However, as shown by Chomiuk \& Povich (2011) the two IMFs give almost identical calibrations.

To test the $L_{\mathrm{ff}}-\mathrm{SFR}$ relation we used the South Pole Telescope (SPT) observations of dusty galaxies at $95 \mathrm{GHz}$ (Mocanu et al., 2013), since we expect that, at this frequency, the free-free emission shows up clearly in local galaxies not hosting a radio loud AGN. To estimate the $95 \mathrm{GHz}$ counts of dusty galaxies Mocanu et al. (2013) adopted a statistical approach. They choose the local minimum in the distribution of the $\alpha_{220}^{150}$ spectral indices, $\alpha_{220}^{150}=1.5$, as the threshold for source classification and computed, for each source, the probability that their posterior $\alpha_{220}^{150}$ is greater 


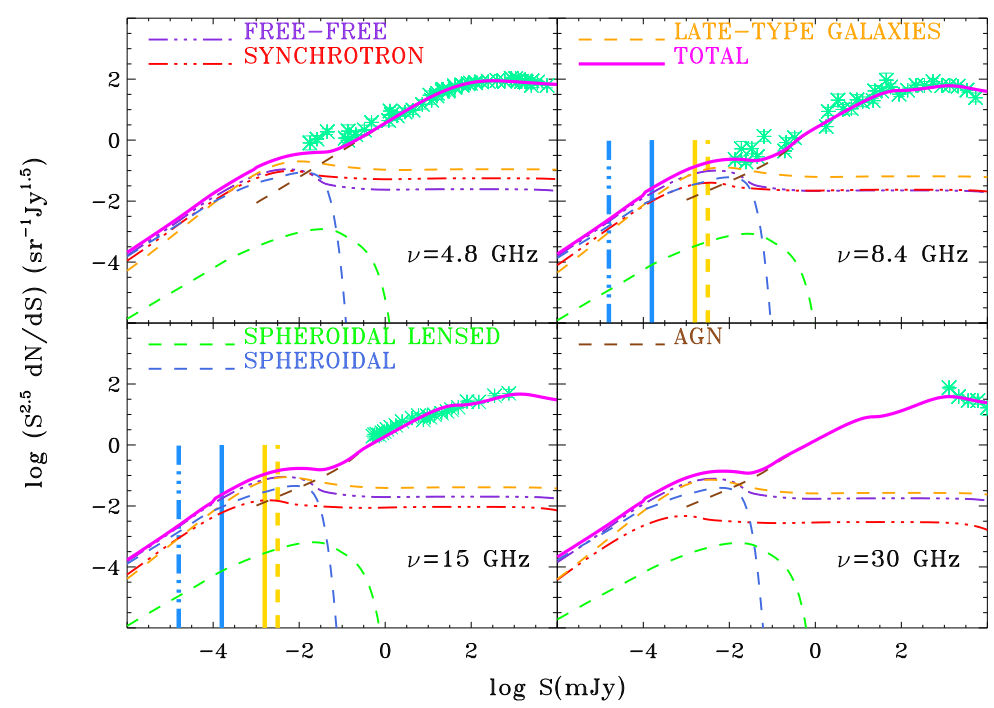

Figure 2: Predicted versus observed counts at 4.8, 8.4, 15 and $30 \mathrm{GHz}$. Dusty galaxies come up at sub-mJy flux density levels and their counts are accounted for by the model. At higher flux densities the counts are dominated by canonical, AGN powered radio sources; the models shown are from Massardi et al. (2010) at 4.8 GHz and from de Zotti et al. (2005) at 8.4, 15 and $30 \mathrm{GHz}$. The solid vertical lines show the limits for predicted deep (yellow) and ultra deep (blue) band 5 SKA1-MID surveys. The dash-dotted blue line and the dashed yellow line are the limits for an Ultra Deep SKA2 (MID) survey and a 50\% SKA1-MID Deep survey respectively.

than the threshold value, $P\left(\alpha_{220}^{150}>1.5\right)$. This quantity was interpreted as the probability that a source is dust-dominated. The $95 \mathrm{GHz}$ differential counts were computed as the sum of probabilities $P\left(\alpha_{220}^{150}>1.5\right)$. Since the fraction of dusty galaxies is much lower than that of synchrotron dominated sources, this statistical approach is endowed with large uncertainties and may strongly overestimate the counts of dusty galaxies.

We have then re-estimated the counts using a lengthier but safer approach, i.e. we have checked the SED of each $95 \mathrm{GHz}$ source brighter than the $95 \%$ completeness limit of $12.6 \mathrm{mJy}$, collecting all the photometric data available in the literature. To model the SEDs we considered both synchrotron and free-free emission, as well as thermal dust emission. We found that only 4 sources, all with $P\left(\alpha_{220}^{150}>1.5\right) \simeq 1$, are indeed dusty galaxies. This is a factor $\simeq 3$ below the number found by Mocanu et al. (2013). The corresponding integral count is shown in the right panel of Fig. 1.

Further tests and/or predictions of the model are provided by counts at 4.8, 8.4,15 and $30 \mathrm{GHz}$ (Fig. 2). The relative importance of free-free compared to synchrotron obviously increases with increasing frequency. While at $1.4 \mathrm{GHz}$ the free-free contribution is always below the synchrotron one, at $8.4 \mathrm{GHz}$ it takes over at tens of $\mu \mathrm{Jy}$ levels.

\section{Predictions for surveys with the Square Kilometer Array}

Preliminary plans for the phase 1 SKA-MID include a set of surveys at $\sim 1-1.4 \mathrm{GHz}$ aimed at investigating the galaxy evolution: an Ultra-Deep survey over $1 \mathrm{deg}^{2}$ with $\mathrm{rms} \sim 50 \mathrm{nJy} / \mathrm{beam}$, a Deep survey over 10-30 $\mathrm{deg}^{2}$ with $\mathrm{rms} \sim 0.2 \mu \mathrm{Jy} /$ beam, and a Wide survey over 1000-5000 


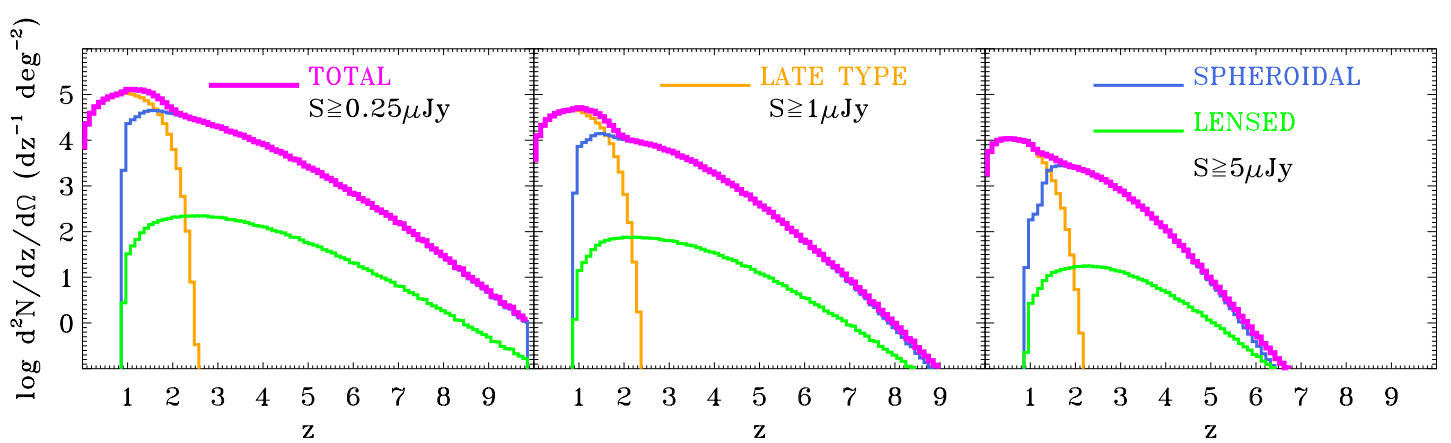

Figure 3: Predicted redshift distributions for $1.4 \mathrm{GHz}$ surveys down to $0.25,1$ and $5 \mu \mathrm{Jy}$, which are the $5 \sigma$ flux density limits of preliminarily planned SKA1-MID surveys.
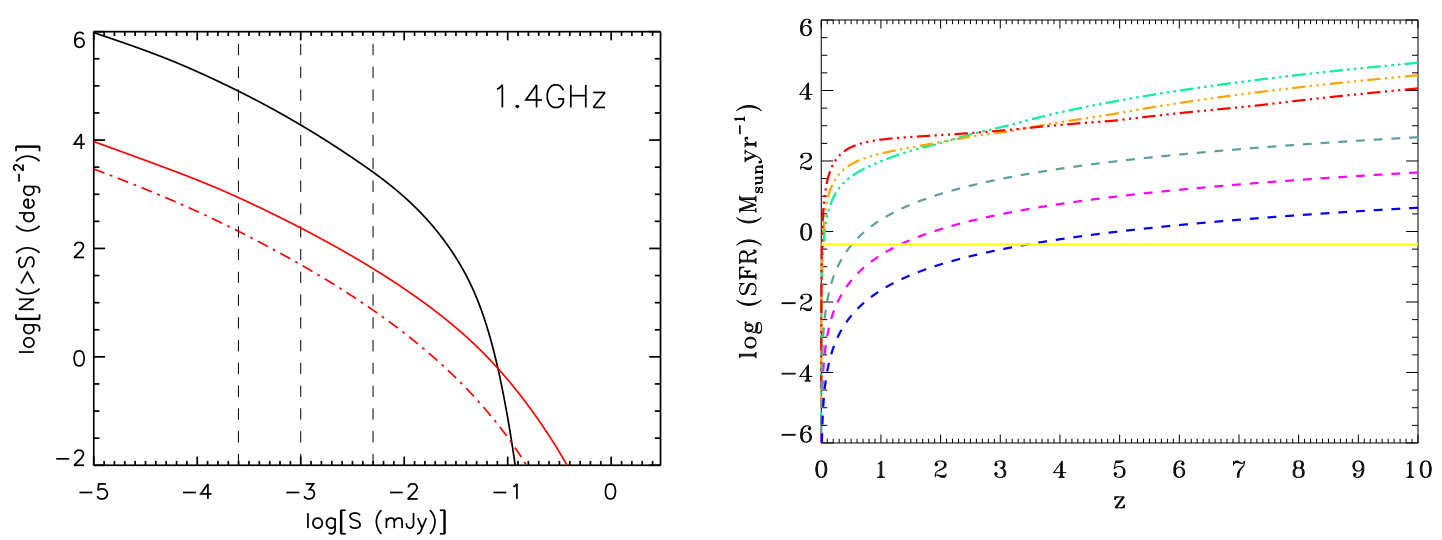

Figure 4: left panel: integral number counts of proto-spheroids at $1.4 \mathrm{GHz}$. Solid black line: unlensed proto-spheroids; solid red line: strongly lensed protospheroids as a function of the total flux density (sum of all images of a single source); dot-dashed red line: counts of strongly lensed proto-spheroids as a function of the flux density of the second brightest image. The vertical dashed lines mark the flux density limits of the possible surveys for SKA1-MID. Right panel: minimum SFR detectable by SKA1-MID (dashed magenta line), 50\% SKA1-MID (dashed light blue line) and SKA2 (MID) (dashed blue line) Ultra-Deep surveys, as a function of $z$, compared with the minimum SFR detected by Herschel surveys (dashed-dot-dot-dot lines, green for $250 \mu \mathrm{m}$, orange for $350 \mu \mathrm{m}$ and red for $500 \mu \mathrm{m}$ ) and by $\mathrm{UV} / \mathrm{H} \alpha$ surveys (horizontal yellow line; see text).

$\operatorname{deg}^{2}$ with rms $\sim 1 \mu \mathrm{Jy} /$ beam (see Dewdney et al. (2013), Braun (2013)). The corresponding $5 \sigma$ limits are indicated by vertical solid lines in the left panel of Fig. 1; the other vertical lines indicate the detection limits achievable with 50\% of SKA1-MID Ultra-Deep and Wide surveys and (full) SKA2 (MID) Ultra-Deep and Wide surveys sensitivity. We also show the contributions to the 1.4 $\mathrm{GHz}$ Euclidean normalized differential number counts of the three populations of dusty galaxies considered by Cai et al. (2013). The main contributors to the "bump" at tens of $\mu \mathrm{Jy}$ levels are late-type galaxies at $z \simeq 1-1.5$. Higher $z$ proto-spheroidal galaxies become increasingly important at lower flux densities, down to a few hundred nJy's.

The predicted redshift distributions for surveys at the SKA1-MID flux density limits are shown in Fig. 3. The fraction of galaxies at very high redshifts $(z \geq 6)$ increases rapidly with decreasing flux density. At a few hundred nJy levels we expect detections of galaxies at $z$ of up to 10, making 
possible to investigate the cosmic SFR across the re-ionization epoch. Note that, although the deepest surveys with the HST are getting close to that, they inevitably miss the dust-obscured star formation, while dust obscuration does not affect SKA measurements.

The fraction of strongly lensed galaxies in flux-limited surveys increases with redshift, to the point that these sources are dominant at the highest redshifts (see Fig. 3). The predicted counts for magnifications $\mu \geq 2$ are illustrated in the left panel of Fig. 4, where the solid black line represents the unlensed proto-spheroidal galaxies, while the red lines represent the lensed galaxies as a function of the total flux density (solid) or of the flux density of the second brightest image (dotdashed). The model predicts 655, 204 and 40 strongly lensed galaxies per $\mathrm{deg}^{2}$ brighter than 0.25 , 1 and $5 \mu \mathrm{Jy}$, respectively; for 250, 100 and 20 of them, respectively, the SKA1-MID will directly detect at least two images. A more detailed discussion on the gravitational lens statistics with SKA is presented in McKean, J., "Strong Gravitational Lensing with the SKA", PoS (AASKA14)084.

The right panel of Fig. 4 compares the SKA potential in measuring the evolution of the cosmic SFR to the outcome of Herschel and of the deepest UV and $\mathrm{H} \alpha$ surveys. The minimum luminosities, hence the minimum SFRs, reached by the latter surveys vary little with redshift. The yellow horizontal line corresponds to their average. Planned SKA surveys can detect galaxies with SFRs from tens to hundred $M_{\odot} / \mathrm{yr}$, up to the highest redshifts, extending the SFR functions by up to 3 orders of magnitude compared with Herschel, thus encompassing SFRs typical of $L_{\star}$ galaxies.

\section{Conclusions}

We have worked out detailed predictions of the counts and redshift distributions for planned SKA surveys, distinguishing the contributions of the different populations of star-forming galaxies: normal late-type, starburst and proto-spheroidal galaxies. The predictions are based on models by Cai et al. (2013) and Cai et al. (2014), that fit a broad variety of UV and far-IR/sub-mm data relevant to determine the epoch-dependent SFR function. These models, however, do not include the contribution to the SFR functions of moderate to low redshift late-type and starburst galaxies. We have upgraded them adding these populations. The upgraded models were combined with the relationships between SFR and radio (synchrotron and free-free) emission derived by Murphy et al. (2011, 2012). Such relationships has been checked exploiting the deepest $1.4 \mathrm{GHz}$ counts and with data from the $95 \mathrm{GHz}$ SPT survey, that we have re-analyzed finding that the published SPT counts of dusty galaxies are overestimated by a factor $\simeq 3$.

We have shown that the SKA will allow us to get information, not affected by dust extinction, on galaxy SFRs down to tens of $M_{\odot} /$ yr up to the highest redshifts, thus extending by up to 3 orders of magnitude the high- $z$ SFR functions derived from Herschel surveys.

\section{Acknowledgements}

We gratefully acknowledge many constructive comments by an anonymous referee, that helped us improving this paper. Work supported in part by ASI/INAF Agreement 2014-024-R.0 for the Planck LFI activity of Phase E2 and by PRIN INAF 2012, project "Looking into the dust-obscured phase of galaxy formation through cosmic zoom lenses in the Herschel Astrophysical Large Area Survey". 


\section{References}

Banday, A. J., Wolfendale, A. W. 1991, MNRAS, 248, 705

Bell, E. F. 2003, ApJ, 586, 794

Bernardi, M., Shankar, F., Hyde, J. B., et al. 2010, MNRAS, 404, 2087

Bonato, M., Negrello, M., Cai, Z.-Y., et al. 2014, MNRAS, 438, 2547

Bourne, N., Dunne, L., Ivison, R. J., et al., 2011, MNRAS, 410, 1155

Bouwens, R. J., Illingworth, G. D., Franx, M., Ford, H. 2008, ApJ, 686, 230

Bouwens, R. J., Illingworth, G. D., Oesch, P. A., et al. 2011, ApJ, 737, 90

Braun, R. 2013, "SKA1 Imaging Science Performance", Document number SKA-TEL-SK0-DDXXX Revision A Draft 2

Cai, Z.-Y., Lapi, A., Bressan, A., et al. 2014, ApJ, 785, 65

Cai, Z.-Y., Lapi, A., Xia, J.-Q., et al. 2013, ApJ, 768, 21

Carilli, C. L., Lee, N., Capack, P., et al. 2008, ApJ, 689, 883

Casey, C. M., Narayanan, D., Cooray, A. 2014, PhR, 541, 45

Chomiuk, L., Povich, M. S. 2011, AJ, 142, 197

Clemens, M. S., Negrello, M., De Zotti, G., et al. 2013, MNRAS, 433, 695

Condon, J. J. 1992, ARA\&A, 30, 575

Dewdney, P., Turner, W., Millenaar, R., et al. 2013, "SKA1 System Baseline Design", Document number SKA-TEL-SK0-DD-001 Revision1

De Zotti, G., Ricci, R., Mesa, D., et al. 2005, A\&A, 431, 893

Draine, B. T. 2011, Physics of the Interstellar and Intergalactic Medium by Bruce T. Draine. Princeton University Press, 2011. ISBN: 978-0-691-12214-4,

Draine, B. T. \& Lazarian, A. 1998, ApJ, 494, L19

Granato, G. L., De Zotti, G., Silva, L., Bressan, A., Danese, L. 2004, ApJ, 600, 580

Helou, G., Soifer, B. T., Rowan-Robinson, M. 1985, ApJ, 298, L7

Helou, G., Bicay, M. D. 1993, ApJ, 415, 93

Hippelein, H., Haas, M., Tuffs, R. J., et al. 2003, A\&A, 407, 137

Ivison, R. J., Swinbank, A. M.;,Swinyard, B., et al. 2010, A\&A, 518, L31

Jarvis, M. J., Smith, D. J. B., Bonfield, D. G., et al. 2010, MNRAS, 409, 92

Kennicutt, R. C., \& Evans, N. J. 2012, ARA\&A, 50, 531

Lacki, B. C, Thompson, T. A., 2010, ApJ, 717, 196

Lapi, A., González-Nuevo, J., Fan, L., et al. 2011, ApJ, 742, 24

Lapi, A., Shankar, F., Mao, J., et al. 2006, ApJ, 650, 42

Mao, M. Y., Huynh, M. T., Norris, R. P., et al. 2011, ApJ, 731, 79

Mao, J., Lapi, A., Granato, G. L., De Zotti, G., Danese, L. 2007, ApJ, 667, 655

Massardi, M., Bonaldi, A., Negrello, M., et al. 2010, MNRAS, 404, 532

McLure, R. J., Dunlop, J. S., Bowler, R. A. A., et al. 2013, MNRAS, 432, 2696

Mocanu, L. M., Crawford, T. M., Vieira, J. D., et al. 2013, ApJ, 779, 61

Murphy, E. J., Bremseth, J., Mason, B. S., et al. 2012, ApJ, 761, 97

Murphy, E. J., Condon, J. J., Schinnerer, E., et al. 2011, ApJ, 737, 67

Murphy, E. J., 2009, ApJ, 706, 482

Niklas, S., Beck, R. 1997, A\&A, 320, 54 
Norris, R. P., Afonso, J., Bacon, D., et al. 2013, PASA, 30, 20

Oesch, P. A., Bouwens, R. J., Illingworth, G. D., et al. 2012, ApJ, 759, 135

Oesch, P. A., Bouwens, R. J., Illingworth, G. D., et al. 2013a, ApJ, 773, 75

Oesch, P. A., Bouwens, R. J., Illingworth, G. D., et al. 2013b, arXiv:1309.2280

Padovani, P., Miller, N., Kellermann, K. I., et al. 2011, ApJ, 740, 20

Planck Collaboration XX 2011, A\&A, 536, A20

Planck Collaboration XVI 2013, arXiv:1303.5076

Schenker, M. A., Robertson, B. E., Ellis, R. S., et al. 2013, ApJ, 768, 196

Smit, R., Bouwens, R. J., Franx, M., et al. 2012, ApJ, 756, 14

Weiß, A., De Breuck, C., Marrone, D. P., et al. 2013, ApJ, 767, 88

Yun, M. S., Reddy, N. A., Condon, J. J. 2001, ApJ, 554, 803 